$\mathrm{O}-\mathrm{\square}$

\title{
Energy and Direction Resolved Secondary Electron Imaging Using Fountain Detector
}

\author{
Takashi Sekiguchi, ${ }^{1, *}$ Toshihide Agemura, ${ }^{1}$ and Hideo Iwai ${ }^{2}$ \\ ${ }^{1}$ Univiersity of Tsukuba, Tenodai 1-1-1, Tsukuba 305-8573, Japan \\ ${ }^{2}$ National Institute for Materials Science, Sengen 1-2-1, Tsukuba 305-0047, Japan \\ *corresponding author's e-mail: sekiguchi.takashi.fp@u.tsukuba.ac.jp
}

(Received: May 30, 2019; Accepted: June 27, 2019)

\begin{abstract}
We have been advocating so-called "Fountain Detector (FD)" for low energy secondary electron (SE) detection in scanning electron microscope (SEM). FDs are simple but easily expanded not only energy filtered but also angle filtered SE image detection. In this presentation, we review our results of energy filtered images of p-n junction of $\mathrm{SiC}_{\text {and }} \mathrm{Si}_{3} \mathrm{~N}_{4}$ nanostructures. Then, we show the recent design of plain angle / directional filter for standard fountain detector and its performance.
\end{abstract}

\section{Introduction}

Recent development of low-voltage scanning electron microscopy (LV-SEM) open up the new field of material science.[1] Since the low energy electron beam interact with the subsurface region of specimen, LV-SEM is sensitive to the surface potential, surface oxidation, contamination etc. In this field, the detection of secondary electrons (SEs) and/or backscattered electrons (BSEs) are also or more important to analyze LV-SEM images. The modern SEMs possess various detectors to select electrons with certain range of energies and directions. Unfortunately, it is difficult to know the collection characteristics of SE/BSE detectors since the lens designs are not disclosed by the SEM providers. To overcome this difficulty, we propose a universal energy and angle resolved SE detector named "Fountain Detector (FD)".[2] Fig. 1a shows the schematics of FD, which is composed of a bias grid between objective lens and specimen, and an electron detector beneath the specimen. The upward SEs with low energies are reflected by the negative bias $-V_{B}$ applied on the grid, going downward to be detected by the detector. By changing the $-\mathrm{V}_{\mathrm{B}}$, we can filter the SEs with their energies. Alternatively, if some area of detector or bias grid is masked, the angle or direction of SEs are also restricted to act as angle/direction selective filter.
We have been developing this FD to improve the filtering performance. By using the spherical grid instead of planar one (Fig. 1b), the energy selection has significantly improved.

We will review several results obtained by this improved FD system.

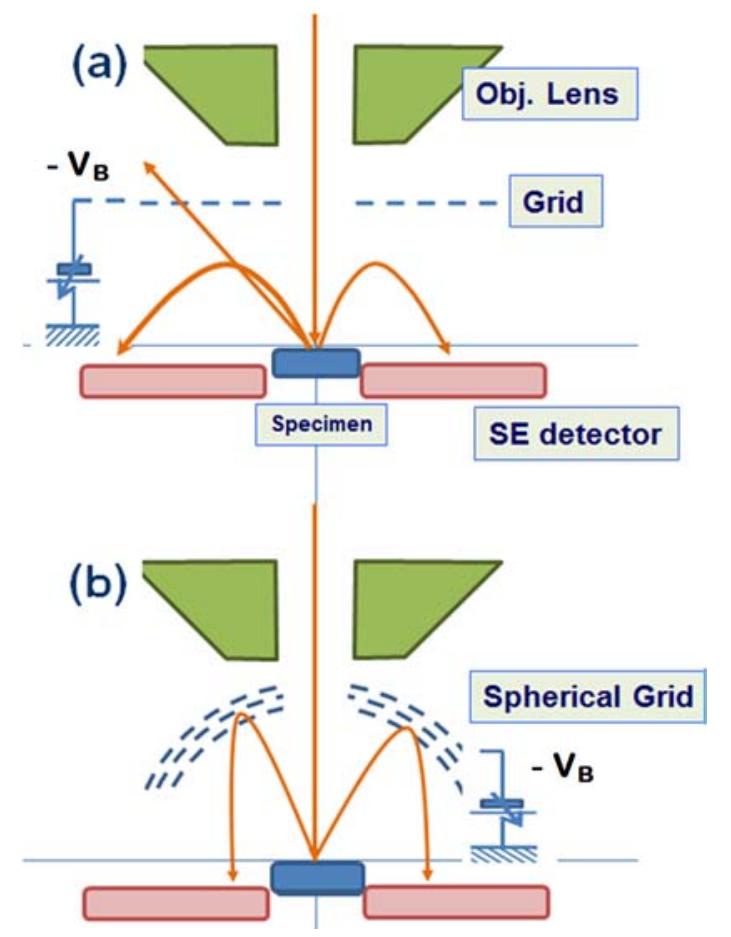

Fig. 1 (a) Schematic of basic Fountain Detector and (b) its improved one using 3 spherical grids. 


\section{2. p-n junction imaging}

The example is p-n junction observation. Most of previous reports said that the p-type region is brighter than the n-type region in SE image. [3]

Fig. 2 shows the filtered FD images of p-n junction in $\mathrm{SiC}$. The image changes drastically with $-\mathrm{V}_{\mathrm{B}}$. When $\mathrm{V}_{\mathrm{B}}>2 \mathrm{eV}$, the p-region is brighter than n-region. This result is consistent with the old report. When $-0.5<-V_{B}$ $<-2 \mathrm{eV}$, however, the p-region is darker than n-region. This indicates that the SE signal intensity varies with its energy. Detailed consideration of band diagram gives the reasonable explanation of this behavior. [4]

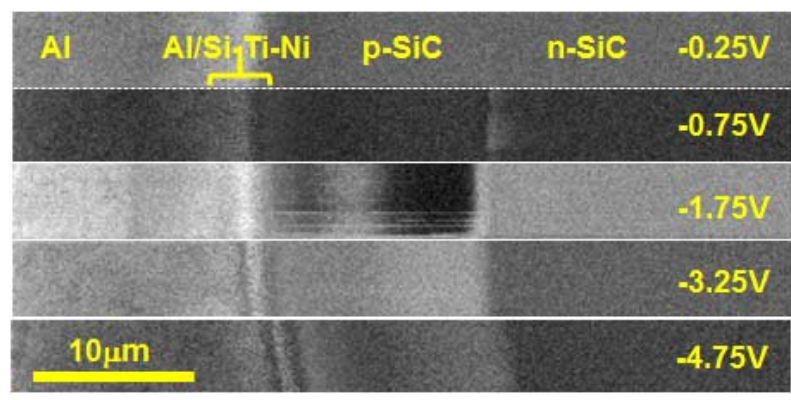

Fig. 2 Energy filtered SE images taken from pn-junctions in SiC. $-V_{B} \mathrm{~S}$ are shown in the right side.

\section{Plain angle / directional filtering for FD}

Recently, we have tried to restrict the angle or direction by inserting mask plate just on the SE detector. The ring mask is used for angle filter, while the sector one for directional filter. The sector mask is effective to enhance the topographic contrast.

\section{References}

[1] M. Nagoshi, K. Sato and T. Aoyama, J. Surf. Anal. 24, 129 (2017).

[2] T. Sekiguchi and H. Iwai, Jpn. J. Appl. Phys. 54, 088001 (2015).

[3] D. D. Perovic, M. R. Castell, A. Howie, C. Lavoie, T. Tiedje, and J. S. W. Cole, Ultramicroscopy 58, 104 (1995).

[4] T. Agemura and T. Sekiguchi, Jpn. J. Appl. Phys. 57, 046701 (2018). 